\title{
The clinical import of a retinal cholesterol embolus: it is a question of symptoms
}

This article was published in the following Dove Press journal:

Clinical Optometry

30 April 2013

Number of times this article has been viewed

\author{
Paul Varner \\ John J Pershing VA Medical Center, \\ Poplar Bluff, MO, USA
}

Correspondence: Paul Varner John J Pershing VAMC,

I500 N Westwood Boulevard,

Poplar Bluff, MO 6390I, USA

Tel +l 5737784303

Fax + I 5737784164

Email paul.varner@va.gov

\begin{abstract}
Retinal cholesterol emboli (RCE) are occasionally found on ophthalmic examination. Originally, all RCE were thought to be related to impending cardiovascular morbidity and mortality, but it is now apparent that accompanying symptoms can be used to help better stratify cardiovascular event risk for these patients. Regardless of associated visual symptoms, all patients with RCE but unknown medical status, merit referral to primary care providers for further assessment of cardiovascular health status. Symptomatic patients with known cardiovascular diseases require carotid duplex ultrasonography to determine degree of arteriolar occlusion with possible referral to vascular surgeons for invasive correction of carotid artery occlusive disease. Asymptomatic patients with known cardiovascular profiles may benefit from the addition of only $81 \mathrm{mg}$ aspirin if not otherwise contraindicated. Screening carotid studies should not be considered for these patients. Carotid auscultation in the eye clinic setting is unwarranted for either set of patients.
\end{abstract}

Keywords: retina, embolus, hollenhorst plaque

\section{Introduction}

Retinal cholesterol emboli (RCE, "Hollenhorst plaques") are atheromatous, refractile cholesterol crystals found within retinal arterioles, often at the site of vessel bifurcation, and are sporadically seen in ophthalmic practice. Large, population-based studies suggest a prevalence of less than $2 \%{ }^{1,2}$ with a 10 -year cumulative incidence of $1 \%-3 \% 0^{3,4}$ in patients essentially over 50 years of age. These same studies confirmed earlier associations between RCE and cardiovascular risk factors, namely, hypertension, smoking, and hypercholesterolemia, although not diabetes or obesity. The independent, clinical implication of these large studies of similar design conducted in different countries is that the presence of RCE is an important marker for stroke prevention and mortality.

The association between RCE and cardiovascular disease has been recognized since Hollenhorst's original paper on the eponymous plaques in 1961. ${ }^{5}$ Subsequent clinical questions addressed the relationship between the presence of RCE and the risk of associated mortality from vascular occlusive disease, but recent studies have focused on medical work-up after discovery of RCE. One critically important distinction that has emerged from this corpus is an assessment of RCE within the context of patient visual symptomatology. ${ }^{6}$

It should be emphasized that cholesterol embolism is not confined to retinal vasculature. Postmortem data suggest a $2 \%$ prevalence of spleen/renal cholesterol emboli in subjects over 60 years of age, although without apparent correlation between 
the emboli and cause of death. ${ }^{7}$ Thus the finding of endorgan cholesterol emboli may not always imply a high risk of morbidity and mortality.

Although there is no definitive consensus on subsequent medical work-up once RCE are discovered, it now appears that medical work-up may be based upon visual symptoms associated with RCE.

\section{Relationship of RCE to morbidity/mortality}

In the 1973 seminal paper on this topic, Pfaffenbach and Hollenhorst reported follow-up data from an initial case series of 208 patients found to have RCE. ${ }^{8}$ Of these participants, a "significant percentage" reported symptoms in conjunction with the finding of RCE. After 5-10 years of follow-up, $65 \%$ of the cohort was deceased, with the vast majority of those deaths (83\%) attributed to vascular disease. Interestingly, the patients in this group were more likely to die from coronary artery disease (mainly myocardial infarction, [MI]) than stroke (cerebrovascular accident, [CVA]) by a margin of $4: 1$. The final recommendation was that the discovery of RCE merits full cardiovascular evaluation.

The authors did not specifically address the finding that RCE are more likely to cause MI than CVA; however, they did address symptomatology, and acknowledged that "whether these data apply to patients who have only the embolus but no other signs or history of vascular disease is unknown"8 (ie, visually asymptomatic patients with indeterminate systemic cardiovascular status). A link between RCE and subjective visual symptoms was not addressed when assigning mortality risk for RCE.

To address this question, Bruno et al studied vascular outcomes in a 1995 cohort study of 70 men with asymptomatic RCE. ${ }^{9}$ During this follow-up period, annual rates for CVA and MI were found to be $8.5 \%$ and $7.7 \%$, respectively, compared to the control group, and the overall mortality rate was not greatly dissimilar between study patients and controls. Unlike the earlier 1973 report, there was no difference between rates of CVA and MI.

Based on these and the earlier findings, it must be inferred that symptomatology is, in and of itself, a significant risk factor in cardiovascular outcomes among patients found with RCE.

\section{Patient visual symptoms}

The first diagnostic consideration after diagnosis of RCE is to query the presence of visual symptoms. From the standpoint of risk stratification, clinical differentiation of RCE depends on associated ocular symptoms. Asymptomatic RCE are entirely incidental exam findings and are unknown to the patient. Symptomatic RCE manifest in two primary ways: amaurosis fugax (fleeting darkness) or central or peripheral vision loss (although in most cases it would be expected that a history of central vision loss need not be elicited).

Monocular amaurosis fugax is the result of transient retinal ischemia, and in the setting of RCE, a manifestation of a partially-occluded arteriole. Retinal ischemia is transitory if RCE initially cause partial occlusion, and then dislodge and move deeper into the vascular arterial plexus. Symptoms of this sort may be recurrent, but are temporary events, and visual function returns to baseline. In essence, amaurosis fugax related to RCE is an ophthalmic transient ischemic attack (TIA).

Permanent loss of visual function occurs if RCE obstruction of the arteriole is complete or long-lived. Arteriolar occlusion of either the central retinal artery or one of its branches results in lasting visual acuity or visual field loss, respectively. Retinal arteriolar occlusion is essentially an ophthalmic CVA. At this time there is no proven treatment for retinal arteriolar occlusion. (It is also important to mention in passing that isolated, unilateral disk edema is not an embolic event and its associations and work-up vary considerably from RCE).

\section{Medical work-up for asymptomatic RCE}

Combined data from the Beaver Dam Eye Study (BDES, $\mathrm{n}=4926$ ) and Blue Mountains Eye Study (BMES, $\mathrm{n}=3654$ ) showed that incidental detection of RCE was associated with a mild increase in all-cause and stroke-related mortality that was independent of other cardiovascular risk factors. ${ }^{10}$ These studies investigated primarily Caucasian populations, but the conclusions are supported by findings from The Los Angeles Latino Eye Study (LALES, $n=5959) .{ }^{11}$ Independent confirmation from these three large population-based studies may be generalizable to all patient groups.

BDES recommendations for follow-up after discovery of asymptomatic RCE are: referral for cardiovascular work-up, smoking cessation, and use of aspirin, but not necessarily carotid endarterectomy. ${ }^{2}$ BMES also suggested alerting primary care providers for consideration of cardiovascular management and smoking cessation, ${ }^{12}$ and these measures are reiterated by the LALES. ${ }^{11}$

Even in the absence of patient symptoms, medical work-up for RCE is indicated for patients with undetermined cardiovascular health status. Agreement among these trials is especially important because these recommendations are made in the context of asymptomatic RCE. 


\section{Utility of carotid ultrasound for asymptomatic RCE}

While cardiovascular assessment appears to be a consensus recommendation, controversy surrounds routine carotid ultrasound, vascular imaging studies (cardiac/aorta), and carotid endarterectomy in asymptomatic patients. ${ }^{13}$ Contradictory evidence has suggested ${ }^{14}$ or disproved ${ }^{15}$ a link between asymptomatic RCE and ipsilateral carotid stenosis, and when performed - carotid imaging reveals $>75 \%$ stenosis in $<20 \%$ of asymptomatic patients with RCE. ${ }^{14,16}$

Questions of carotid artery occlusive disease are more often discussed in the literature of vascular surgeons, ie, those performing the surgical interventions. More than 20 years of vascular surgery literature has not changed: the presence of asymptomatic RCE is a poor marker for significant carotid artery occlusive disease ${ }^{17-21}$ Fortunately, some ophthalmic literature is in agreement that the discovery of asymptomatic RCE should not be used to guide clinical decision-making for ultrasonography, to determine atherosclerotic disease of the carotid arteries. ${ }^{3,22}$

Given the limitations of useful clinical information from carotid studies for asymptomatic RCE, it does not appear that duplex ultrasonography should be routinely obtained for this isolated clinical finding. This finding is in contrast to earlier recommendations. ${ }^{6}$

\section{Utility of carotid auscultation in the setting of asymptomatic RCE}

Carotid auscultation is the procedure for listening for a bruit caused by carotid stenosis. Bruits are the result of turbulent hemodynamic flow through stenotic lesions and can develop once arterial lumen is reduced to less than $50 \% .{ }^{23}$ Although the presence of a carotid bruit has been associated with an increased risk of cerebrovascular disease,${ }^{24}$ this clinical finding is also coupled with poor predictive value for confirmatory Doppler duplex scanning ( $43 \%)$ and confounded by the finding that one-third of patients with a bruit have normal ultrasounds (false positives, with respect to bruits) ${ }^{25}$ Conversely, one-third of patients with high-grade carotid stenosis manifest no bruit (false negatives with respect to bruits). ${ }^{26}$

As the finding of bruit is clinically most useful in symptomatic patients, ${ }^{23}$ employment of carotid auscultation in the presence of asymptomatic RCE must be objectively questioned by clinicians.

\section{Is any intervention indicated for asymptomatic RCE?}

The North American Symptomatic Carotid Endarterectomy Trial (NASCET) demonstrated the efficacy of carotid endarterectomy in symptomatic patients (with 70\%-99\% occlusion) ${ }^{27}$ however, the benefit of carotid surgery in the presence of an asymptomatic RCE is unclear. ${ }^{28}$

Aspirin has been shown to decrease cardiovascular events in patients at risk, ${ }^{29,30}$ although the clinical utility of daily aspirin based on incidental finding of asymptomatic RCE is unknown. Together with medical management of existing cardiovascular disease and modification of cardiovascular risk factors (eg, smoking cessation, decreasing serum cholesterol level, controlling systemic hypertension, maintaining an active lifestyle, healthy diet), daily aspirin therapy is perhaps the only intervention required after diagnosis of asymptomatic RCE. The efficacy of other antiplatelet modalities also remains unknown.

It should also be emphasized that $81 \mathrm{mg}$ of aspirin achieves the same therapeutic antiplatelet effect as $325 \mathrm{mg}$ tablets, but with a lower side effect profile. ${ }^{31}$ The decision to initiate daily aspirin therapy is ultimately one of risk stratification and perhaps best made in the primary care setting.

\section{RCE diagnosed through teleretinal screening programs}

As the use of telemedicine screening programs for eye diseases increases, diagnosis of incidental ophthalmic findings will correspondingly escalate. In practice, remote identification of RCE is commensurate with diagnosis on direct retinal examination and carries the same outcomes after empiric medical work-up (ie, identification of cardiovascular disease, limited findings for carotid studies). ${ }^{16}$ Once RCE are identified on retinal photos, a telephone call replaces face-to-face history taking in order to determine the presence of visual symptoms. The same work-up applies, as for direct clinical encounters. Also, as retinal emboli can persist for more than one year, ${ }^{17}$ it may be possible to view the same RCE in sequential images.

\section{Summary of clinical questions}

Table 1 provides a summary of findings from this literature review.

Table I Summary of clinical questions to consider after the diagnosis of an asymptomatic retinal cholesterol embolus

\begin{tabular}{ll}
\hline Is medical work-up indicated? & Yes \\
Is carotid ultrasound indicated? & No \\
Is carotid auscultation useful? & No \\
Is any intervention indicated? & $81 \mathrm{mg}$ aspirin daily, when \\
& not contraindicated \\
Is diagnosis through teleretinal & No, contact patient and \\
screening program different? & query symptoms \\
\hline
\end{tabular}




\section{Conclusion}

RCE are in vivo markers of atheromatous disease, and clinical prognosis depends largely on associated visual symptomatology. RCE in the presence of transient or permanent visual acuity or visual field loss require carotid studies to determine patient candidacy for carotid endarterectomy. Asymptomatic RCE appear to be relatively benign ocular findings. This is not to imply that asymptomatic RCE should be lightly regarded; they represent abnormal findings, originate from atheromatous sources, and denote active cardiovascular disease. However, beyond referral for basic cardiovascular work-up, ancillary testing is likely unnecessary. Incidental discovery of asymptomatic RCE merits referral to primary care for assessment and modification of cardiovascular risk factors. In the absence of contraindications, these patients may be started on low-dose aspirin daily for antiplatelet effect. An algorithm for RCE may be found in Figure 1. Due to confounding findings, carotid auscultation is not necessarily indicated in the presence of any RCE.

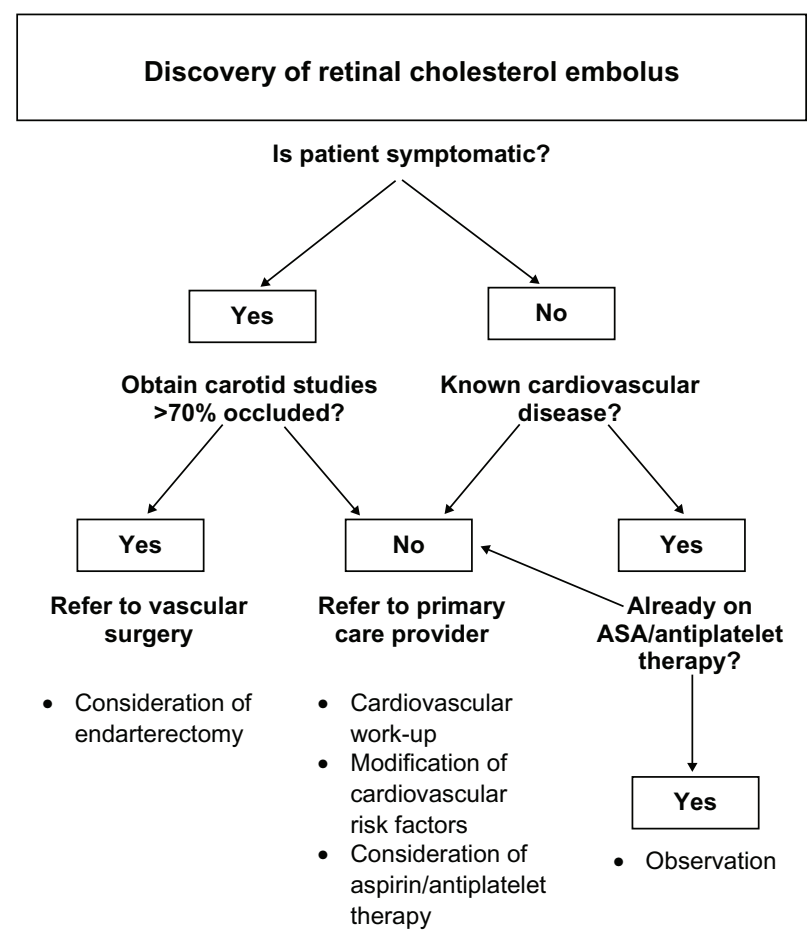

Figure I Flowchart for work-up of retinal cholesterol emboli (RCE).

\section{Acknowledgment}

The author would like to thank Antonia Varner for her assistance in the preparation of the flowchart.

\section{Disclosure}

The views expressed in this article are those of the author and do not necessarily represent the position of the US Department of Veterans Affairs. The author reports no proprietary or commercial conflicts of interest in this work.

\section{References}

1. Mitchell P, Wang JJ, Weining L, et al. Prevalence of asymptomatic retinal emboli in an Australian urban community. Stroke. 1997;28: 63-66.

2. Klein R, Klein BEK, Jensen SC, et al. Retinal emboli and stroke: the Beaver Dam eye study. Arch Ophthalmol. 1999;117:1063-1068.

3. Klein R, Klein BEK, Moss SE, Meuer SM. Retinal emboli and cardiovascular disease: the Beaver Dam eye study. Trans Am Ophthalmol Soc. 2003;100:173-182.

4. Cugati S, Wang JJ, Rochtchina E, Mitchell P. Ten-year incidence of retinal emboli in an older population. Stroke. 2006;37:908-910.

5. Hollenhorst RW. Significance of bright plaques in the retinal arterioles. JAMA. 1961;178:23-29.

6. Coutu R. Treatment and management of the Hollenhorst plaque. Clin Eye Vis Care. 1993;5:52-58.

7. Cross SS. How common is cholesterol embolism? J Clin Pathol. 1991:44:859-861.

8. Pfaffenbach DD, Hollenhorst RW. Morbidity and survivorship of patients with embolic cholesterol crystals in the ocular fundus. Am J Ophthalmol. 1973;75:66-72.

9. Bruno A, Jones WL, Austin JK, et al. Vascular outcomes in men with asymptomatic retinal cholesterol emboli: a cohort study. Ann Intern Med. 1995;122:249-253.

10. Wang JJ, Cugati S, Knudtson MD, et al. Retinal arteriolar emboli and long-term mortality: pooled data analysis from two older populations. Stroke. 2006;37:1833-1836.

11. Hoki SL, Varma R, Lai MY, et al. Prevalence and associations of asymptomatic retinal emboli in Latinos: the Los Angeles Latino eye study. Am J Ophthalmol. 2008;145:143-148.

12. Mitchell P, Wang JJ, Smith W. Risk factors and significance of finding asymptomatic retinal emboli. Clin Experiment Ophthalmol. 2000;28:13-17.

13. Biousse V. Cerebrovascular disease. In: Miller NR, Newman NJ, Biousse V, Kerrison JB, editors. Walsh and Hoyt's Clinical Neuro-Ophthalmology, 6th ed. Philadelphia: Lippincott Williams \& Wilkins; 2004;2.

14. O'Donnell BA, Mitchell P. The clinical features and associations of retinal emboli. Aust N Z J Ophthalmol. 1992;20:11-17.

15. Bruno A, Russell PW, Jones WL, et al. Concomitants of asymptomatic retinal cholesterol emboli. Stroke. 1992;23:900-902.

16. Ahmed R, Khetpal V, Merin LM, Chomsky AS. Case series: retrospective review of incidental retinal emboli found on diabetic retinopathy screening: is there a benefit to referral for work-up and management? Clin Diabetes. 2008;26:179-182.

17. Bunt TJ. The clinical significance of the asymptomatic Hollenhorst plaque. J Vasc Surg. 1986;4:559-562.

18. Schwarcz TH, Eton D, Ellenby MI, et al. Hollenhorst plaques: retinal manifestations and the role of carotid endarterectomy. J Vasc Surg. 1990;11:635-641.

19. Wakefield MC, O’Donnell SD, Goff JM Jr. Re-evaluation of carotid duplex for visual complaints: who really needs to be studied? Ann Vasc Surg. 2003;17:635-640.

20. McCullough HK, Reinder CG, Hynan LS, et al. Ocular findings as predictors of carotid artery occlusive disease: is carotid imaging justified? J Vasc Surg. 2004;40:279-286.

21. Dunlap AB, Kosmorsky GS, Kashyap VS. The fate of patients with retinal artery occlusion and Hollenhorst plaque. J Vasc Surg. 2007;46: $1125-1129$. 
22. Sharma S, Brown GC, Pater JL, Cruess AF. Does a visible retinal embolus increase the likelihood of hemodynamically significant carotid artery stenosis in patients with acute retinal artery occlusion? Arch Ophthalmol. 1998;116:1602-1606.

23. Sandercock PAG, Kavvadia E. The carotid bruit. Pract Neurol. 2002;2:221-224.

24. Pickett CA, Jackson JL, Hemann BA, Atwood JE. Carotid bruit and cerebrovascular disease risk: a meta-analysis. Stroke. 2010;41: 2295-2302.

25. Davies KN, Humphrey PRD. Do carotid bruits predict disease of the internal carotid arteries? Postgrad Med J. 1994;70:433-435.

26. Sauve JS, Thorpe KE, Sackett DL, et al. Can bruits distinguish high-grade from moderate symptomatic carotid stenosis? The North American symptomatic carotid endarterectomy trial. Ann Intern Med 1994;120:633-637.
27. North American Symptomatic Carotid Endarterectomy Trial Collaborators. Beneficial effect of carotid endarterectomy in symptomatic patients with high-grade carotid stenosis. N Engl J Med. 1991;325:445-453.

28. Wolintz RJ. Carotid endarterectomy for ophthalmic manifestations: is it ever indicated? J Neuroophthalmol. 2005;25:259-261.

29. British Cardiac Society, British Hyperlipidaemia Association, British Hypertension Society, British Diabetic Association. Joint British recommendations on prevention of coronary heart disease in clinical practice: summary. BMJ. 2000;320:705-708.

30. US Preventive Services Task Force. Aspirin for the prevention of cardiovascular disease: US Preventive Services Task Force recommendation statement. Ann Intern Med. 2009;150:396-404.

31. Campbell CL, Smyth S, Montalescot G, Steinhubl SR. Aspirin dose for the prevention of cardiovascular disease: a systematic review. JAMA 2007;297:2018-2224.

\section{Clinical Optometry}

\section{Publish your work in this journal}

Clinical Optometry is an international, peer-reviewed, open access journa publishing original research, basic science, clinical and epidemiologica studies, reviews and evaluations on clinical optometry. All aspects of patient care are addressed within the journal as well as the practice of optometry including economic and business analyses. Basic and clinical

\section{Dovepress}

research papers are published that cover all aspects of optics, refraction and its application to the theory and practice of optometry. The manuscript management system is completely online and includes a very quick and fair peer-review system, which is all easy to use. Visit http://www.dovepress. $\mathrm{com} /$ testimonials.php to read real quotes from published authors. 